\title{
DETAILED BALANCE IN REACTION KINETICS - CONSEQUENCE OF MASS CONSERVATION?
}

\section{Miloslav Pekař}

Institute of Physical and Applied Chemistry, Faculty of Chemistry, Brno University of Technology, Purkyňova 118, 61200 Brno, Czech Republic

Phone: +420-541149330

Fax: $+420-541211697$

E-mail: pekar@fch.vutbr.cz

Detailed balance condition for triangular isomerisation mechanism is derived from the more general permanence of atoms, consequently, it should be viewed as a special equilibrium result of mass conservation.

Keywords: detailed balance, kinetics and thermodynamics, mechanism

\section{INTRODUCTION}

Detailed balance is a useful principle that has been intuitively used by generations of chemists as already Onsager [1] noted in perhaps the first communication that analyzed this principle. The principle of detailed balance states that overall equilibrium of a chemical reaction cannot be achieved without equilibration of all its steps [2]. To the best of our knowledge there is no macroscopic or phenomenological proof or derivation of the detailed balance. The principle is explained on molecular level by another principle - the microscopic reversibility [3].

Recently, Alberty [4] recalled the role of triangular mechanism 


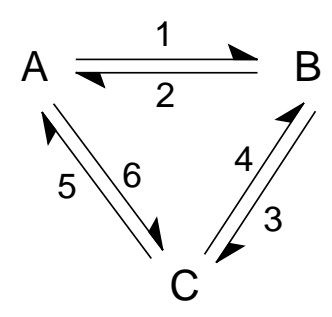

in development and illustration of the detailed balance. Mechanism (1) represents an isomerisation reaction. As potential candidates, isomerisations of $x y-$ lene [5], cymene [6] or butene [7, 8] can be given. Alberty [4] reminds that the detailed balance leads to the following restriction on the rate constants $\left(k_{i}\right)$ in the triangle mechanism (1):

$$
k_{1} k_{3} k_{5}=k_{2} k_{4} k_{6}
$$

Here, it will be shown for the first time that the detailed balance condition (2) is a result of permanence of atoms, in other words, of mass conservation. The permanence of atoms is closely related to the idea of independent chemical reactions, which, in fact, is the key point of the proof. Therefore, it is further shown how the interconversion of isomers A, B, C can be treated consistently with modern irreversible thermodynamics. The derivation given here is more general than traditional justification of detailed balance because it is not restricted to equilibrium conditions only.

\section{RESULTS AND DISCUSSION}

The initial presumption is nothing more than the classical kinetic mass-action law, which is also hidden in condition (2). Then, the rates $\left(r_{i}\right)$ of the three steps from mechanism (1) are given by:

$$
\begin{aligned}
& r_{1}=k_{1} c_{\mathrm{A}}-k_{2} c_{\mathrm{B}} \\
& r_{2}=k_{3} C_{\mathrm{B}}-k_{4} C_{\mathrm{C}} \\
& r_{3}=k_{5} c_{\mathrm{C}}-k_{6} c_{\mathrm{A}}
\end{aligned}
$$


where $c_{i}$ is the concentration of isomer $i$ and $k_{j}$ are the rate constants. Bowen [9] showed how the permanence of atoms, i.e., the conservation of mass, can be treated by linear algebra. Using his treatment of chemically reacting systems it is found that in a system of three isomers only two independent reactions (and their rates) are possible. Consequently, one of the three reaction rates is a function or combination of the other two. Let us select the third reaction rate as the dependent one, than generally:

$$
r_{3}=a_{1} r_{1}+a_{2} r_{2}
$$

where $a_{1}, a_{2}$ are the coefficients of linear combination to be determined. Introducing the mass-action expressions, Eq. (3), it results:

$$
\left(a_{1} k_{1}+k_{6}\right) c_{\mathrm{A}}+\left(a_{2} k_{3}-a_{1} k_{2}\right) c_{\mathrm{B}}+\left(-a_{2} k_{4}-k_{5}\right) c_{\mathrm{C}}=0
$$

Equation (5) is an identity and should be valid for any concentration values, hence the coefficients in parentheses should vanish. From the first and third we obtain:

$$
a_{1}=-k_{6} / k_{1} ; a_{2}=-k_{5} / k_{4}
$$

and from the second

$$
a_{2} k_{3}=a_{1} k_{2}
$$

Introducing Eq. (6) into Eq. (7), Eq. (2) is recovered.

Detailed balance and independence of reactions thus impose the same restriction on the values of rate constants in the mass-action rate equations. They are just two ways of formulating the permanence of atoms. However, the detailed balance operates on equilibrium only whereas the derivation based on the permanence of atoms is free of this restriction and therefore valid in general. Thus, the detailed balance can be considered, on phenomenological level, just as a special equilibrium consequence of the mass conservation in chemical reactions. 
Alberty showed [4] that batch reactor balance equations for mechanism (1) with the rates given by Eq. (3) can be solved numerically even when the rate constants do not fulfil the condition 2 and quite acceptable concentration profiles can be obtained. The solution obtained with parameters violating Eq. (2) may be acceptable in the sense of giving positive concentrations and finishing in a state of no concentration changes. In other words, experimental data need not directly detect a violation of the detailed balance conditions and can be well fitted by traditional rate equations not fulfilling that condition. Modern thermodynamics offers much more consistent description.

Samohýl introduced an interesting approach to the design of rate equations [10-14] that is based on methods and results of the rational thermodynamics [15]. He proved that in a mixture of fluids with linear transport properties, the reaction rate is a function of temperature and concentrations only, $r=r\left(T, c_{1}, \ldots\right.$, $c_{n}$ ). The fluids with linear transport properties cover most fluid systems of chemists' interest and can be viewed as fluids with viscosity, heat transport, diffusion conforming to traditional laws like the Newton viscosity, Fourier heat, Fick diffusion laws. Only the independent reactions are considered. The rate function is approximated by a polynomial of suitable degree in concentrations.

The first order polynomial approximation to the rate function of three isomers $(\mathrm{A}, \mathrm{B}, \mathrm{C})$ reacting mixture reads:

$$
\mathbf{r}=\overline{\mathbf{k}}_{0}+\overline{\mathbf{k}}_{1} C_{\mathrm{A}}+\overline{\mathbf{k}}_{2} C_{\mathrm{B}}+\overline{\mathbf{k}}_{3} C_{\mathrm{C}}
$$

Bold letters denote vectors; in this case two independent reactions are possible and their rates (denoted here by $\bar{r}_{1}, \bar{r}_{2}$ ) are the components of the vector $\mathbf{r}$ : $\mathbf{r}=\left(\bar{r}_{1}, \bar{r}_{2}\right)$. The polynomial coefficients $\overline{\mathbf{k}}_{i}$ are also two-component vectors, generally: $\overline{\mathbf{k}}_{i}=\left(\bar{k}_{i, 1}, \bar{k}_{i, 2}\right)$ where suffices 1 and 2 refer to the first and second independent reaction, resp. 
Selecting the first and second steps of mechanism (1) as the independent reactions and applying equilibrium condition [10-14] on Eq. (8), the following equation is derived:

$$
\mathbf{r}=\overline{\mathbf{k}}_{1}\left(c_{\mathrm{A}}-K_{1}^{-1} C_{\mathrm{B}}\right)+\overline{\mathbf{k}}_{3}\left(c_{\mathrm{C}}-K_{2} C_{\mathrm{B}}\right)
$$

where $K_{i}$ is the equilibrium constant of independent step $i$. In this approach, both independent reactions have the same rate equation but with generally different values of polynomial coefficients $\left(\overline{\mathbf{k}}_{i}\right)$, which are then interpreted as rate constants. The component formation rates $\left(R_{i}\right)$ are given by material balance and stoichiometry as:

$$
R_{\mathrm{A}}=-\bar{r}_{1}, R_{\mathrm{B}}=\bar{r}_{1}-\bar{r}_{2}, R_{\mathrm{C}}=\bar{r}_{2}
$$

for instance:

$$
\begin{aligned}
& R_{\mathrm{B}}=\left(\bar{k}_{1,1}-\bar{k}_{1,2}\right)\left(c_{\mathrm{A}}-K_{1}^{-1} c_{\mathrm{B}}\right)+\left(\bar{k}_{3,1}-\bar{k}_{3,2}\right)\left(c_{\mathrm{C}}-K_{2} c_{\mathrm{B}}\right) \\
& R_{\mathrm{C}}=\bar{k}_{1,2}\left(c_{\mathrm{A}}-K_{1}^{-1} c_{\mathrm{B}}\right)+\bar{k}_{3,2}\left(c_{\mathrm{C}}-K_{2} c_{\mathrm{B}}\right)
\end{aligned}
$$

Selecting $\quad \bar{k}_{1,1}=k_{1}^{\prime}>0, \quad \bar{k}_{3,2}=-k_{2}^{\prime}<0, \quad \bar{k}_{1,2}=\bar{k}_{3,1}=0$, the traditional massaction rate equations are obtained:

$$
\begin{aligned}
& r_{1}^{\prime}=k_{1}^{\prime}\left(c_{\mathrm{A}}-K_{1}^{-1} C_{\mathrm{B}}\right) \\
& r_{2}^{\prime}=k_{2}^{\prime}\left(K_{2} C_{\mathrm{B}}-c_{\mathrm{C}}\right)
\end{aligned}
$$

Figure 1 shows a comparison of modelling outputs for Alberty's case III [4] calculated with the Eq. (3) (points) with the outputs obtained with the rational thermodynamic alternative approach (lines), Eq. (12). The rate constants for the case III are: $k_{1}=2, k_{2}=k_{3}=k_{4}=k_{5}=k_{6}=1$ [4] and violate the principle of detailed balance. The values of constants used for the alternative approach were: $k_{1}^{\prime}=3.00, k_{2}^{\prime}=7.36, K_{1}=1.68, K_{2}=0.79$. Clearly, data which would be fitted in the traditional approach, not considering (in)dependence of reactions, 
with constants violating the detailed balance (or permanence of atoms) can be well fitted by the alternative approach, which is free of such defects. In other words, unrealistic data obtained with the triangle mechanism rate equations are very close to quite realistic data calculated with the two-step mechanism.

Note, that the illustrated alternative approach is just a replacement of the three-step mechanism 1, composed from dependent reactions, by the set of two independent reactions $\mathrm{A}=\mathrm{B}, \mathrm{B}=\mathrm{C}$. The rates of the two steps can be given, e.g., by Eq. (9) or (12). Interestingly, studying xylene isomerization Allen and Yats found [5] that one of the triangular steps 1 (viz. o- and p-xylene interconversion) actually does not occur and has effectively zero rate constants. Thus, their experiments proved that this reacting mixture of three isomers is described by the two independent steps only. This was confirmed also by Miklósy et al. [16]. The experiments on cymene isomerization revealed [6] that the reaction steps are not first order or pseudo-first order. Mechanism of cymene isomerization therefore does not correspond to the scheme 1 exactly.

Of course, the alternative two-step approach based just on Eq. (12) does not give a non-zero production rate of $\mathrm{C}$ in the time zero (exactly) if the initial concentrations of B and C are zero. On the other hand, the triangular mechanism 1 can predict, in principle, a non-zero C production under these conditions. However, it is very difficult if not impossible to determine experimentally reaction rate exactly in the zero time or the zero time itself. Anyway, the general alternative description by Eq. (9), i.e., without the special selection of rate constants made before Eq. (12) above, allows a non-zero initial formation rate for $\mathrm{C}$ even when $c_{\mathrm{B}}^{0}=c_{\mathrm{C}}^{0}=0$, cf. general Eq. (11b). Further, presented analysis should not mean that the triangular mechanism is a non-sense and should be abandoned. If this mechanism is applied, however, then the condition (2) must be fulfilled; if not, this is an indication that the reaction course is more complex (e.g. some in- 
termediates have not been revealed). Butene isomerization seems to occur according to the triangle mechanism and to fulfil the condition $(2)[7,8,17]$.

The whole analysis is strictly valid for a mixture of just the three isomers, i.e. no other components (e.g. intermediates) exist, and for the first order transformations. Only the condition (4) has a general validity in this mixture. It can be easily checked that the condition (2) remains valid if all reaction steps are of the same order.

\section{CONCLUSIONS}

Detailed balance condition for the triangular isomerisation mechanism has been derived from the more general permanence of atoms, not restricted to equilibrium only. The detailed balance can be then viewed as a special result of the mass conservation at equilibrium. The permanence of atoms, which is equivalent to the mass conservation, restricts the number of independent reactions. Consequently, the triangular mechanism has been shown to be adequately transformable to and representable by a simpler two-step version, containing only independent reactions, satisfactorily fitting experimental data.

\section{REFERENCES}

1. L. Onsager: Phys. Rev., 37: 405 (1931).

2. K. Denbigh: Thermodynamics of the Steady State. Methuen, London 1951.

3. R.C. Tolman: The Principles of Statistical Mechanics. Oxford University Press, Oxford 1939.

4. R.A. Alberty: J. Chem. Educ., 81: 1206 (2004).

5. R.H. Allen, L.D. Yats: J. Am. Chem. Soc., 81: 5289 (1959).

6. R.H. Allen, T. Alfrey, L.D. Yats: J. Am. Chem. Soc., 81: 42 (1959).

7. W.O.Haag, H.Pines: J. Am. Chem. Soc., 82: 387 (1960).

8. D. Kalló: J. Catal., 66: 1 (1980). 
9. R.M. Bowen: Arch. Rational Mech. Anal., 29: 114 (1968).

10. I. Samohýl: Collection Czechoslov. Chem. Commun., 40: 3409 (1975).

11. I. Samohýl, A. Malijevský: Collection Czechoslov. Chem. Commun., 41: 2131 (1976).

12. I. Samohýl: Rational Thermodynamics of Chemically Reacting Mixtures (in Czech). Academia, Prague 1982.

13. I. Samohýl: Thermodynamics of Irreversible Processes in Fluid Mixtures. Teubner, Leipzig 1987.

14. M. Pekař: Chem. Eng. Sci., 59: 4103 (2004).

15. C. Truesdell: Rational Thermodynamics. McGraw-Hill, New York 1984.

16. É. Miklósy, J. Papp, D. Kalló: Zeolites, 3: 139 (1983).

17. J.Wei, C.D. Prater: in Advan. Catal. (D.D. Eley, P.W. Selwood, and P.B. Weisz, eds.), 13: 203, Academic Press, New York and London 1962.

This work was partially supported by the Czech government funding - project MSM 0021630501. 
Figure 1. Plots of concentrations of isomers A, B, and C versus time calculated with three dependent (points; Eq. 3; A - box, B - circle, C - diamond) or two independent (lines; Eq. 12) reaction steps. For parameters used in calculations see text.

Figure 1

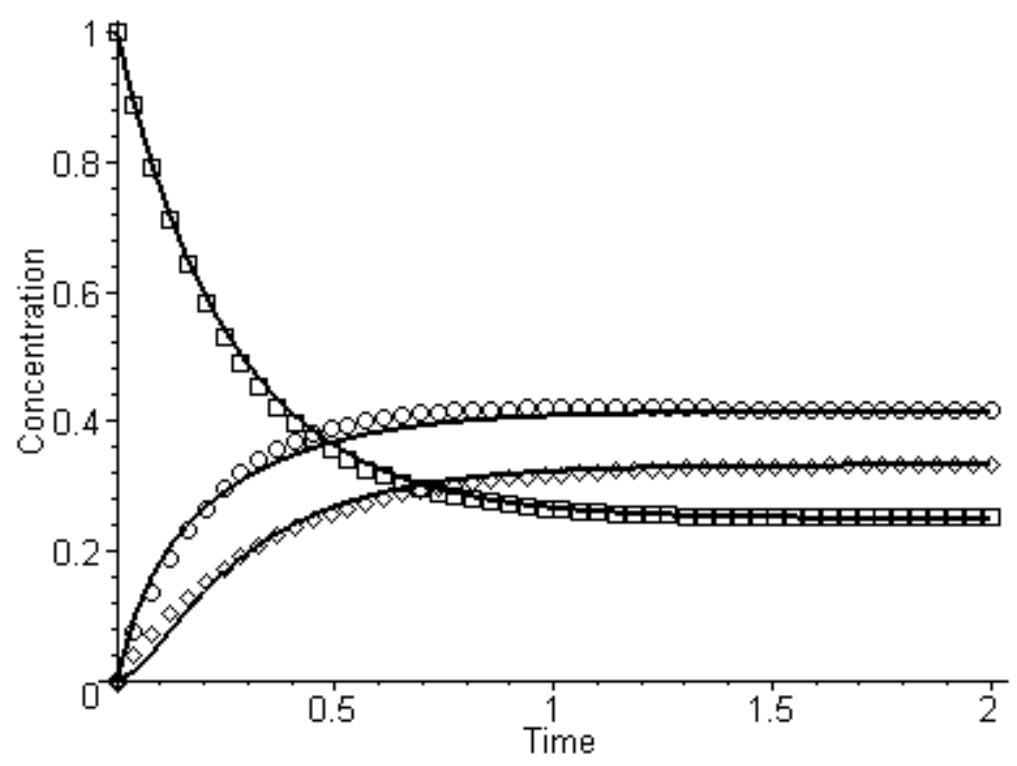

